\title{
The HiSPARC-UK Cosmic Ray Detector
}

\section{Cristina Lazzeroni}

University of Birmingham

School of Physics and Astronomy, Edgbaston B15 2TT, UK

E-mail: c. lazzeroni abham.ac.uk

\section{Maria Pavlidou ${ }^{1}$}

University of Birmingham

School of Physics and Astronomy, Edgbaston B15 2TT, UK

E-mail: m.pavlidou@bham.ac.uk

\section{Angela Romano}

University of Birmingham

School of Physics and Astronomy, Edgbaston B15 2TT, UK

E-mail: angela.romano@cern.ch

\section{Antonino Sergi}

University of Birmingham

School of Physics and Astronomy, Edgbaston B15 2TT, UK

E-mail: antonino.sergiecern.ch

The international HiSPARC project is a collaboration between schools and academic institutions across Europe in real scientific research, and won the European Physical Society HEP outreach prize in 2007. It was born more than a decade ago in the Netherlands, with ongoing participation of over 100 schools. Since 2011, HiSPARC has been introduced to the United Kingdom and we report on its successful local story.

EPS-HEP2017, European Physical Society conference on High Energy Physics 5-12 July 2017

Venice, Italy

\section{${ }^{1}$ Speaker}




\section{Introduction}

The HiSPARC project [1] is an international collaboration of secondary schools and academic institutions for high energy cosmic ray air shower research and public engagement. It consists of a network of detectors measuring high energy cosmic ray showers (Fig 1), and won the European Physical Society HEP outreach prize in 2007. It was born more than a decade ago in the Netherlands, with active participations of more than 100 schools. Relatively recently, HiSPARC has been introduced to the UK, with a network of schools forming mainly in the Midlands/South West.

HiSPARC-UK started its growth in the Bristol area as part of the cosmic ray centenary, with support from the South-West HE-STEM, AWE, the UK Institute of Physics and the University of Bristol. The project expanded to the Birmingham area in 2014 with support from the University of Birmingham and the Ogden Trust. In 2016 the network expanded to Wales (Cardiff \& Swansea), with a grant from the Welsh Assembly. The UK network has thus increased rapidly, and currently has 27 schools and support from the Science and Technology Facility Council, and is part of the Institute for Research in Schools initiative [2].

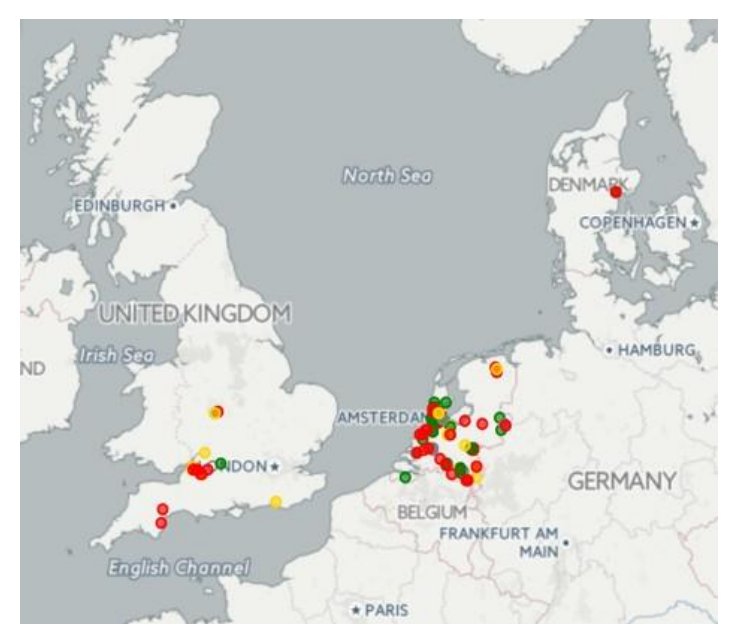

Fig 1: HiSPARC Network detector

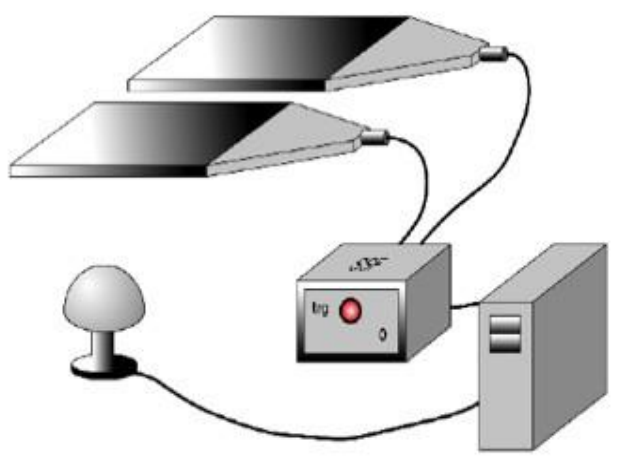

Fig 2: Schematic of HiSPARC detector

\section{The HiSPARC detector in schools}

Schools joining the network are provided with components for a rooftop, researchquality, cosmic ray detector [3]. This comprises a pair of scintillators and phototubes, and an Internet-connected ADC / GPS unit that uploads data to a central database (Fig. 2). A typical detector configuration includes two scintillators inside weather-proof containers, while a configuration with four scintillators allows the determination of the shower direction. Students are responsible for the detector assembly and installation and the monitoring of its performance (Fig 3). The schools then maintain and operate the 
detector over several years, passing the task to generations of students. Students have therefore the opportunity to gain valuable knowledge of particle physics detector techniques and methods and exploit our academic research links to CERN and our longstanding expertise in detectors as well as in particle and astroparticle research and analysis of the collected data.
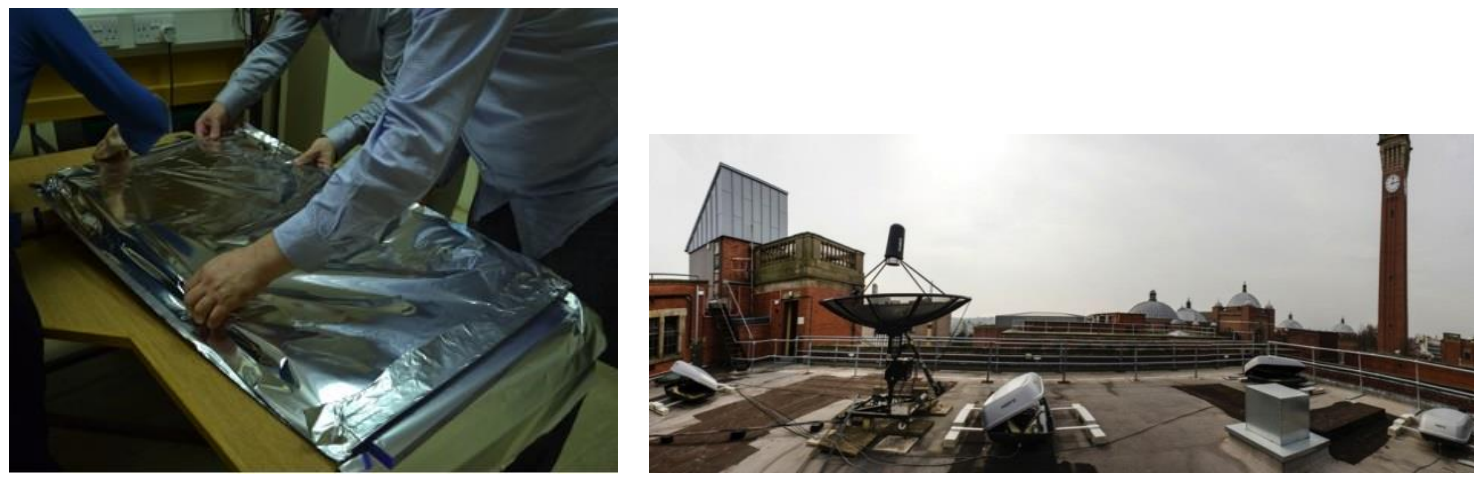

Fig 3: Detector construction, and position on roof

\section{Data analysis}

Data are read-out automatically into a central database; the web interface provides a simple way to access monitor histograms and basic data, while the entirety of raw data can be downloaded for each detector station (Fig 4). Students initially discuss with researchers possible specific research projects that range from studying the influence of latitude or altitude on shower development, to the determination of shower core energy and position (Fig 5). Typically each school groups will choose a broad research topic and teams will research specific ideas independently. The link with researchers is maintained via visits or remote video links on a regular basis, to follow the students' progress. Results are shared and discussed at an annual School Conference where students give presentations to their peers.

Station: 14001 - Birmingham University

Tue, 27 June 2017

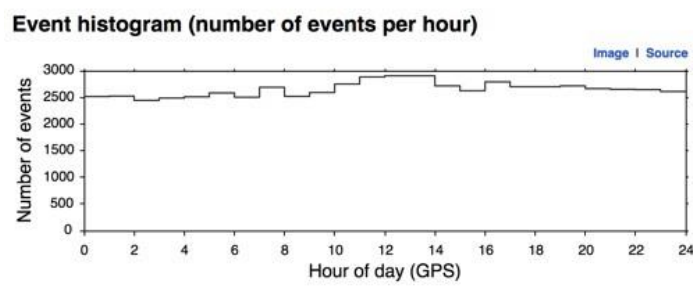

Fig 4: HiSPARC data

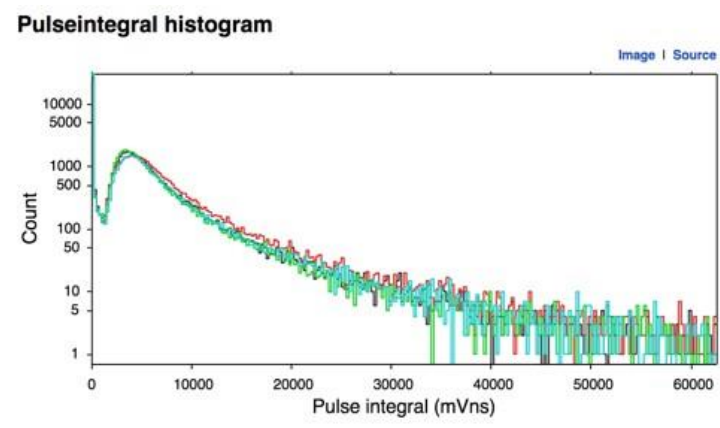


The apparatus allows research-quality science to be performed in schools, thanks to the access to the entire HiSPARC database. Therefore students gain valuable knowledge of particle physics detector techniques and methods and exploit our academic research links to CERN (Birmingham, Bristol, Sussex), as well as having access to cutting-edge investigations like the upper limit on the energy of cosmic rays from distant sources (GZK cut-off), detection of widely separated, nearly simultaneous events (GerasimovaZatsepin effect) and triangulation of very high energy cosmic rays, thus exploiting our other academic research links in astrophysics and astroparticle physics (Bath, Leeds, Exeter).
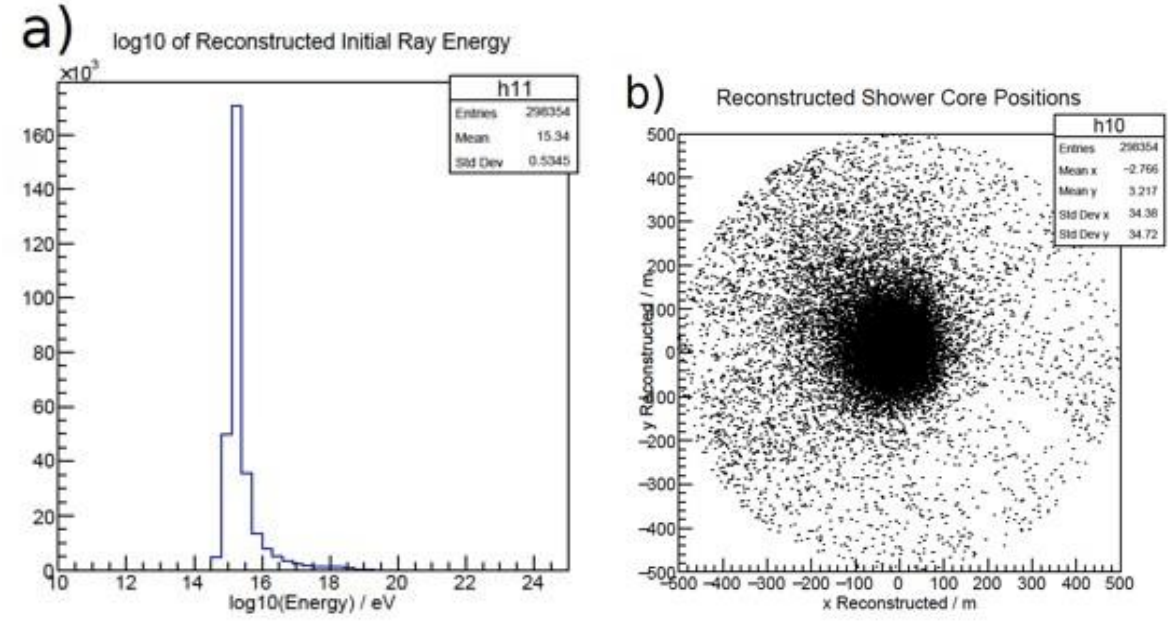

Fig 5: Reconstructed shower energy and position

\section{Evaluating impact}

We have observed that students fully engage with the HiSPARC project, thanks to the cutting-edge aspect of the research and the general research environment, including the annual conference where they discuss their progress and results with fellow students and researchers in a truly research-oriented and inspiring environment. The HiSPARC project also boosts research interactions and networking between the academic partners, and between the teachers in the participating schools. HiSPARC naturally fosters longterm collaborations between schools and academic partners, therefore enhancing the impact on students. This aspect, when combined with practical as well as speculative aspects of the research, is particularly beneficial for engaging with female students and students from underprivileged areas $[5,6]$.

The impact of the project is best demonstrated looking at a particular female school located in an under-developed area of Birmingham with a significant ethnic population. The HiSPARC project had a tremendous impact on the 12 girls that took part in it in 2014-15, scientifically and personally. The majority of the girls involved have changed their perception of the subject and decided to progress to university studies in a STEM subject. The school managed to attract 2 prestigious educational prizes because of the 
project and the school management is committed to continue working with HiSPARC for years to come.

\section{Conclusions}

HiSPARC is a successful international project in the area of particle astrophysics that aims to take cutting-edge cosmic ray shower science into secondary schools, therefore exploiting the links between public engagement and research.

\section{References}

[1] HiSPARC home page: http://www.hisparc.nl

[2] IRIS home page: www.researchinschools.org

[3] D. Fokkema, "The HiSPARC Experiment"; PhD thesis

[4] M. Stankaityte, Lab Y3 Student Project @ University of Birmingham

[5] http://link.springer.com/article/10.1007/s11165---008---9083---Z

[6] http://www.kcl.ac.uk/sspp/departments/education/research/ASPIRES/ASPIRES--final---report---December---2013.pdf 